Title: MULTIPLE-SHOCK INITIATION VIA STATISTICAL CRACK MECHANICS

$$
\text { CONF-980803-- }
$$

Author(s):

Submitted to:

John K. Dienes

Detonation Theory \& Application, Group T-14

Los Alamos National Laboratory

James D. Kershner

Detonationa Theory \& Application, Group T-14

Los Alamos National Laboratory

The 11th International Detonation Symposium,

Snowmass, Colorado, August 31-September 4, 1998

DISTRIBUTON OF THS DOCVRENT IS UARLBMTED MASTER
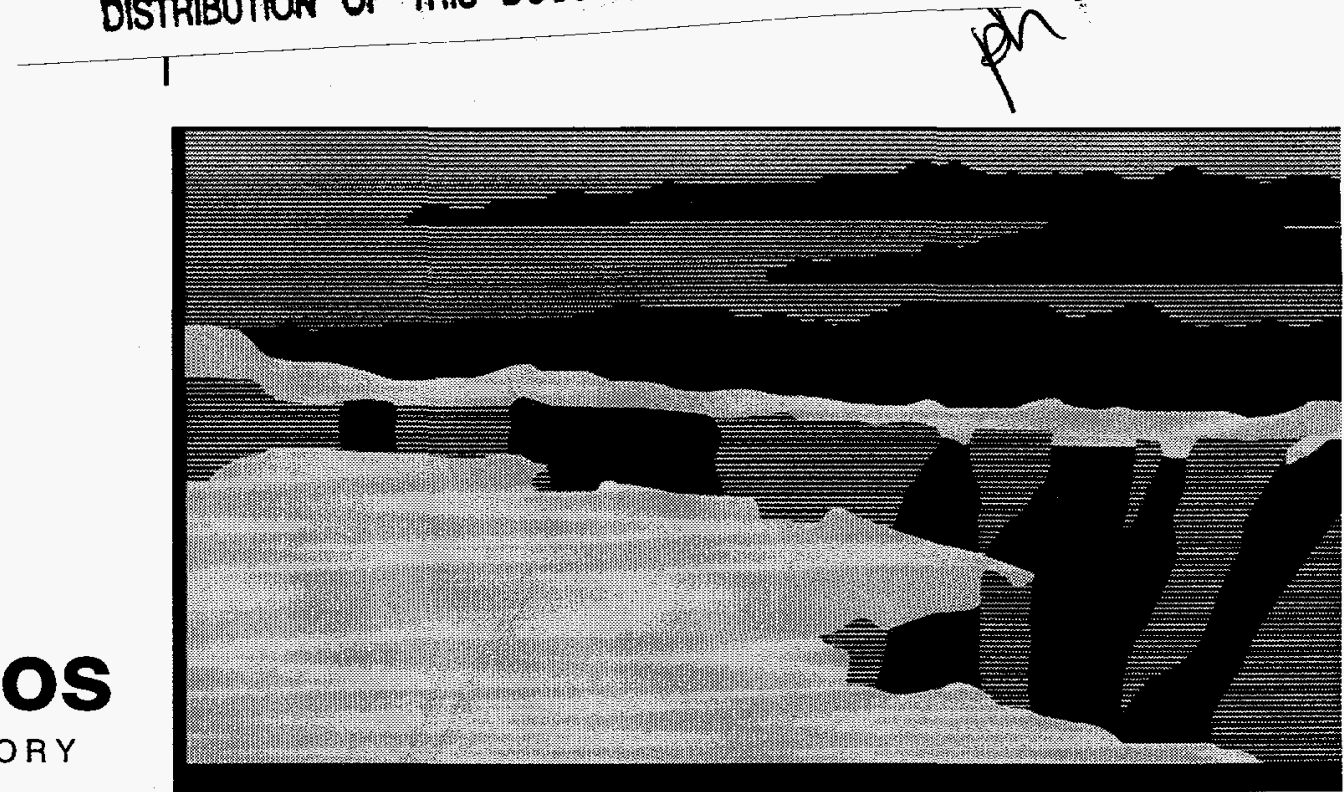

Los Alamos National Laboratory, an affirmative action/equal opportunity employer, is operated by the University of California for the U.S. Department of Energy under contract W-7405-ENG-36. By acceptance of this article, the publisher recognizes that the U.S. Government retains a nonexclusive, royalty-free license to publish or reproduce the published form of this contribution, or to allow others to do so, for U.S. Government purposes. The Los Alamos National Laboratory requests that the publisher identify this article as work performed under the auspices of the U.S. Department of Energy. 


\section{DISCLAIMER}

This report was prepared as an account of work sponsored by an agency of the United States Government. Neither the United States Government nor any agency thereof, nor any of their employees, makes any warranty, express or implied, or assumes any legal liability or responsibility for the aceuracy, completenesa, or usefulness of any information, apparatus, product, or process dieclosed, or represents that its use would not infringe privately owned rights. Reference herein to any specific commercial product, process, or service by trade name, trademark, imanufacturer. or otherwise does not necessarily constitute or imply its endorsenent, recommendation. of favoring by the United States Governmeat or any ageacy thereof. The views and opinions of authors expressed herein do not necessarily state or reflect those of the United States Government or any agency thereof. 


\section{DISCLAIMER}

Portions of this document may be illegible in electronic image products. Images are produced from the best available original document. 


\title{
MULTIPLE-SHOCK INITIATION VIA STATISTICAL CRACK MECHANICS
}

\author{
J.K. Dienes and J.D. Kershner \\ Theoretical Division, Los Alamos National Laboratory \\ Los Alamos, NM 87545
}

\begin{abstract}
Statistical Crack Mechanics (SCRAM) is a theoretical approach to the behavior of brittle materials that accounts for the behavior of an ensemble of microcracks, including their opening, shear, growth, and coalescence. Mechanical parameters are based on measured strain-softening behavior. In applications to explosive and propellant sensitivity it is assumed that closed cracks act as hot spots, and that the heating due to interfacial friction initiates reactions which are modeled as onedimensional heat flow with an Arrhenius source term, and computed in a subscale grid. Postignition behavior of hot spots is treated with the burn model of Ward, Son and Brewster. Numerical calculations using SCRAM-HYDROX are compared with the multiple-shock experiments of Mulford et al. in which the particle velocity in PBX 9501 is measured with embedded wires, and reactions are initiated and quenched.
\end{abstract}

\section{INTRODUCTION}

Brittle materials fail in a manner quite different from ductile materials, with growth and coalescence of cracks being the main features. We have shown that this behavior can be described by direct modeling of cracks using the principle of Superposition of Strain Rates which. in fact, holds for a wide variety of physical processes. This allows us to represent combined brittle and ductile behavior, and to incorporate processes such as high-pressure effects into a general treatment of material failure. When applied to explosives and propellants this method allows us to explain both the fluctuations in violence seen in $\mathrm{XDT}^{\mathrm{l}}$ and size effects ${ }^{2}$. It can be generalized as necessary to account for additional mechanical and chemical phenomena.

Since hot spots are initially small, internal, and evolve rapidly, it is not normally possible to observe them directly. Simulations are therefore difficult to verify, but the slow initiation obtained by Mulford et $\mathrm{al}^{3}$ in their multiple-shock experiment gives us an opportunity to confirm the overall behavior. (Von Holle ${ }^{4}$ has observed hot spots on the surface of explosives and Schmitt et $\mathrm{al}^{5}$ have made direct observations of very hot shear bands in shocked quartz, but these are not necessarily related to the internal hot spots in explosives that cause accidents.)

Wiegand has measured the mechanical failure of PBX $9501^{6}$ for a variety of temperatures and strain rates. We have been able to model his results with $\mathrm{SCRAM}^{7,8}$ and from them infer average values of micromechanical parameters such as crack size, surface energy, and number density that are needed in our continuum calculations. This is more straightforward than direct observation of cracks, and less tedious. (It would be of some interest to corroborate the inferred crack properties by direct observation.) Closed cracks can heat up as a result of interfacial friction, and this is thought to initiate reactions in many cases of interest. Other hot-spot mechanisms have been considered ${ }^{9}$, but closed cracks have the requisite size and are considered the most likely candidate for initiation by the authors at this point.

The chemical response to hot-spot formation falls into two regimes. In the first, ignition, the heat equation with a source term is integrated to the point where the peak hotspot temperature is near its critical value. Linan and Williams ${ }^{10}$ have shown the existence of such a critical value, and demonstrated an analytical approach to evaluate it when the heating rate is uniform. Though this is not normally the case, the analytic solution does provide a useful check on our numerical scheme Following ignition, burn is assumed to occur at a rate determined by a special solution to the heat equation obtained by Ward, Son, and Brewster ${ }^{11}$ (WSB) which is based on two-stage chemistry and shows impressive agreement with experiments ${ }^{12,13}$. At higher pressures the faster burn rate described by Forest ${ }^{14}$ is required to match the measurements of Mulford et al. ${ }^{3}$

\section{SUPERPOSITION, CRACK MECHANICS, STATISTICS, AND STRAIN SOFTENING}

Superposition of strain rates was originally introduced as an ansat ${ }^{15}$ in connection with elasticplastic flow, but it is valid for a much greater variety of processes as shown theoretically by Dienes ${ }^{16}$. Formally. it takes the form

$$
\mathbf{D}=\sum \mathbf{D}^{\alpha}
$$

where $\mathbf{D}$ denotes the stretching tensor (the symmetric part of the velocity gradient) and $\mathbf{D}^{\alpha}$ denotes the contribution 
of the $\alpha$ th process. (Stretching and strain rates are actually different quantities ${ }^{16}$, but the distinction is not essential here or within the scope of this paper.) We take the first process as elastic deformation of the damaged material, the second as cracking, the third as plastic flow. and the fourth as the effect of high-pressure nonlinearities. which are taken as isotropic.

The utility of superposition of strain rates as a basis for a constitutive law depends on formulating useful relations for the $\mathbf{D}^{\alpha}$ as functions of stress, stress-rate and possibly other variables of the flow. In the case of the elastic part of the deformation

$$
\mathbf{D}^{1}=\mathbf{C}: \hat{\sigma}
$$

where the compliance $C$ is the sum of the compliance of the matrix material and the contribution from internal microcracks, as discussed in Appendix $A$, and $\hat{\sigma}$ is the polar stress rate ${ }^{16,17}$. (This is the ordinary rate of change of Cauchy stress supplemented by terms that account for material rotation.) The second term involves crack growth, which influences the compliance according to

$$
\mathbf{D}^{2}=\hat{\mathbf{C}}: \sigma
$$

Since the compliance depends only on crack size, c. which is time dependent, for growing cracks $\hat{\mathbf{C}}$ becomes $C_{e} \dot{c}$, where the subscript denotes the derivative with respect to $c$. The rotation implied by the carat is not relevant, and the matrix character of $\hat{\mathbf{C}}$ can be omitted. since a scalar compliance is sufficient to characterize the plane-wave behavior considered herein. The crack speed $\dot{c}$ is a function of the stress intensity factor alone, as discussed in Appendix B.

Some plastic behavior in PBX materials under pressure is thought to take place, but it is not well understood. Dislocations have been observed ${ }^{18}$, and twinning has been seen in HMX crystals, and we are assuming that, when confined ${ }^{19}$. plastic flow occurs at high pressure but the assumption that plastic flow can occur in PBXs has not been verified. As usual, plastic flow is represented in SCRAM by

$$
\mathbf{D}^{3}=\lambda \sigma,
$$

but here $\lambda$ is a scalar parameter that depends on the elastic, fracture, and plastic-flow parameters as derived by Dienes $^{17}$, and given therein as equation 14.91. The nonlinear part of the Mie-Gruneisen equation of state is represented as a function of specific energy $e$ and density $\rho$ in rate form $D^{4}$ (e.p) in order to maintain consistency with other portions of the constitutive description.

The crack statistics are used in Appendix A to develop an expression for the compliance $\boldsymbol{C}$. This involves the distribution function $N(c, \Omega, t)$, which represents the number of isolated cracks with orientation $\Omega$ exceeding $c$ in radius, per unit (initial) volume. In most SCRAM work the crack orientation is defined by one of 9 directions on the unit sphere. This distribution is initially exponential in c. However, the total number of isolated cracks declines with crack growth, while coalescence causes a set of connected cracks with distribution function $M(c, \Omega, t)$ to develop as discussed by Dienes ${ }^{17}$. Cracks are assumed to intersect when their size reaches the mean-free-path. Complete coalescence, after $\alpha$ intersections (statistical data is needed for the variable $\alpha$ ), terminates crack growth. The number of connected cracks in a set is discussed in Appendix $\mathrm{C}$ of Ref 26 . The connected crack sets are what is usually observed, and when they becomes large, at the percolation threshold, large cracks with complex shapes resulting from crack coalescence permeate the material. This percolation threshold is important for, when burning cracks reach this critical point, a violent reaction of the entire mass is expected.

\section{CRACK SPEED, TOUGHNESS, MELTING, AND INERTIA}

The crack speed approaches one third of the speed of sound at high stresses according to data collected by Stroh $^{20}$, while theoretical studies by Freund ${ }^{21}$ conclude that it can approach the Rayleigh wave speed. In the earliest versions of SCRAM, which involved very violent processes, crack speed was assumed constant, but as SCRAM is applied to lower-energy processes, it becomes necessary to account for the reduced speed. This is done by Freund, who derives a formula of the type $v=v_{v}\left(1-K_{0}^{2} / K^{2}\right)$ with the fracture toughness $K$ defined by $K=\sqrt{2 \pi E \gamma}$ ( $E$, Young's Modulus; $\gamma$, surface energy). However, at values of the stress intensity factor $K$ below $K_{0}$, crack speed is thought to follow a power law in $K^{22.23}$. The two regions can be joined in a consistent way by having the curves osculate at the value of $K_{1}$ given by Eq. B.7, as discussed in Appendix B.

Fracture toughness plays an important role in the growth of cracks, but its value is not constant in a composite material. In SCRAM the surface energy is taken as the measure of crack resistance. In PBX 9501 the value of $\gamma$ is about $.05 \mathrm{~J} / \mathrm{m}^{2}$ for cracks within an $\mathrm{HMX}$ grain $^{24}$, but it increases considerably when the crack reaches the binder. Based on fitting the data of Wiegand $^{7.8}$, the surface energy of the composite is $\gamma=120 \mathrm{~J} / \mathrm{m}^{2}$. A simple energy theory, presented in Appendix D, leads to a value of $195 \mathrm{~J} / \mathrm{m}^{2}$.

Melting point is known to play an important role in sensitivity ${ }^{25}$. When the interfacial heating between the faces of a closed crack brings the local temperature to the melting point, the frictional heating on the interface drops, and the heating is considered to become diffuse over the liquid region, which is very thin (sub-micron). Hence, the 
strain rate and the resulting heating can be very high, bringing the local temperature to the ignition point. As the phase transition to melting is being completed, the heating rate is considered to decrease linearly with internal energy, with the flow stress decreasing from the yield stress of the solid to the viscous stress of the liquid.

Crack inertia arises from the kinetic energy of the adjoining material. Values for a penny-shaped crack are computed in Appendix $E$ of the Dienes, Kershner paper ${ }^{26}$ on the assumption that the deformations are those for a static crack, certainly not exact but hopefully a useful approximation. For a stable crack the kinetic energy arises from opening, while for an unstable crack the kinetic energy is enhanced by its rate of growth. The value for virtual mass is used in the equation for crack opening $\mathbf{w}$,

$$
m \ddot{w}+c_{d} \dot{w}+k w=p A
$$

The cavity pressure $p$ is derived from the WSB burn model and the area $A$ is just the area of the penny-shaped crack. No value for the damping $c_{d}$ is available, though an educated guess can be made from a general knowledge of material behavior.

\section{IGNITION - A NUMERICAL ALGORITHM AND LINAN-WILLIAMS THEORY}

Since the heated zone adjacent to a closed crack is normally very thin, it is a useful approximation to consider the heat flow to be one dimensional and normal to the crack plane. The heating is computed only at the center of the penny-shaped crack, where it is maximum. In the IGNC subroutine of SCRAM the governing equation, in the notation of Ref. 17, is taken as

$$
\dot{\mathrm{T}}=\mathrm{DT}_{\mathrm{xx}}+\left(\mathrm{zQ} / \rho \mathrm{C}_{\mathrm{v}}\right) \exp (-\mathrm{E} / \mathrm{kT}),
$$

with the boundary condition $k T_{x}=-\dot{q}$ at $x=0$ when below the melting point. Here $\dot{q}$ represents the frictional heating $\mu \sigma_{n} v$, with $\sigma_{n}$ the normal component of traction, $\mu$ the coefficient of friction, and $v$ the sliding velocity. This velocity is computed from the variable traction on the crack, if it is closed, but is taken to be zero when the crack is open, since then there is no frictional heating. At the melting point and above, the heat flux is taken to be zero, but then the viscous heating is added to the Arrhenius source term. The nonlinear equation shown above is solved numerically by the Crank-Nicholson method. The accuracy of the method and comparisons with published results are given by Dienes ${ }^{27}$. In test calculations it is observed that the temperature rises very rapidly above a certain point. This critical temperature has been investigated by Linan and Williams ${ }^{30}$, who show that there is a definite point where the Arrhenius term becomes important and the subsequent temperature rises rapidly In view of this behavior, it is not necessary to include depletion in the source term, since it matters only when the temperature is above the ignition point. The parameter A of Linan-Williams is found to be $1.03 \mathrm{e} 15$ for a certain set of conditions, given in Appendix $F$ of the Dienes, Kershner paper ${ }^{26}$, while application of the IGNC subroutine gives $\mathrm{A}=0.96 \mathrm{e} 15$. This agreement is considered satisfactory, considering that both calculations are approximate.

Above the ignition point the IGNC subroutine would no longer compute the reaction correctly. A transition to burning is assumed to take place and the burn rate is computed using the results of WSB ${ }^{1 !}$.

\section{BURN}

The literature on burn is far too extensive to be more than touched on here, having been driven by the needs of the propellant industry. We mention only the work of Forest $^{14}$, who was concerned with explosives and Pop plots, and the WSB results mentioned above. Both give results for the bum rate as a function of pressure, which are shown in Figure 1 and are in good agreement. The rapid bum region above $2000 \mathrm{MPa}$ has apparently not been explored by WSB. The burn rate is computed in SCRAM using the numerical method described in Appendix $G$ of the Dienes, Kershner paper ${ }^{26}$. It is of some interest that the method accounts for a wide range of solid temperatures, since the accident scenarios with which we are concerned are most likely at extreme temperatures.

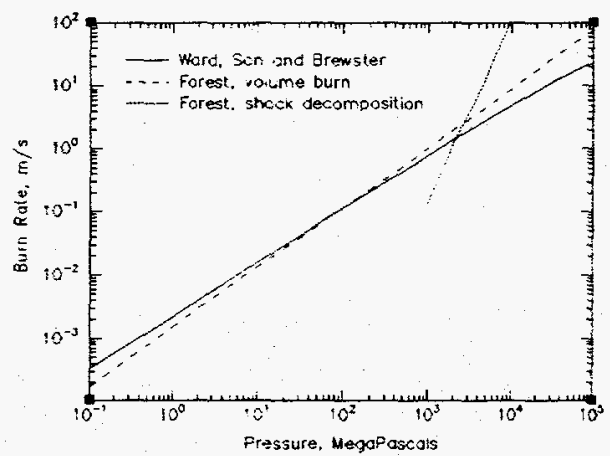

FIGURE 1. BURN RATES AS COMPUTED BY FOREST AND BY WARD, SON AND BREWSTER

\section{HYDROX CALCULATION}

HYDROX $^{28}$ is a one-dimensional shock code that has been used for a wide variety of problems. It was selected for the current multiple-shock analysis in view of its flexibility, convenience, and graphical versatility. The experiment of Mulford et $\mathrm{al}^{3}$ to be studied involves a flyer plate consisting of two materials, first kel-F and then vistal, which cause first and second shocks to propagate into the PBX 9501 target. The material properties for the three materials are listed in Appendix $\mathrm{H}$ of the Dienes. Kershner paper ${ }^{26}$. The flyer-plate is represented usin - the 
standard HYDROX material model, while the PBX 9501 is represented using SCRAM.

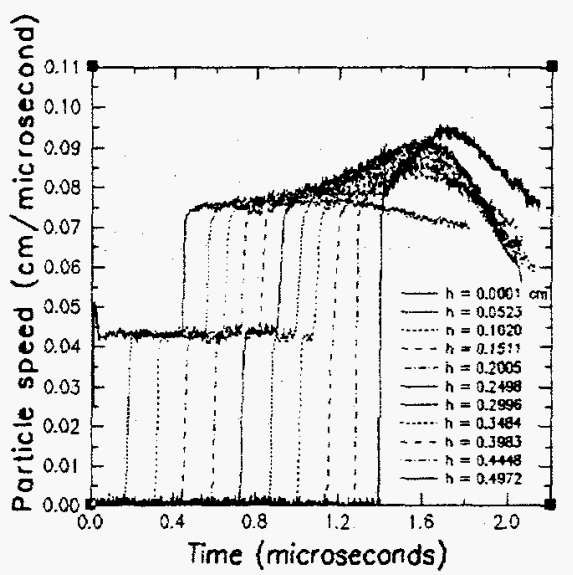

FIGURE 2. MULTIPLE SHOCK EXPERIMENT SHOWING VELOCITIES AT 11 LOCATIONS AS MEASURED BY EMBEDDED WIRES

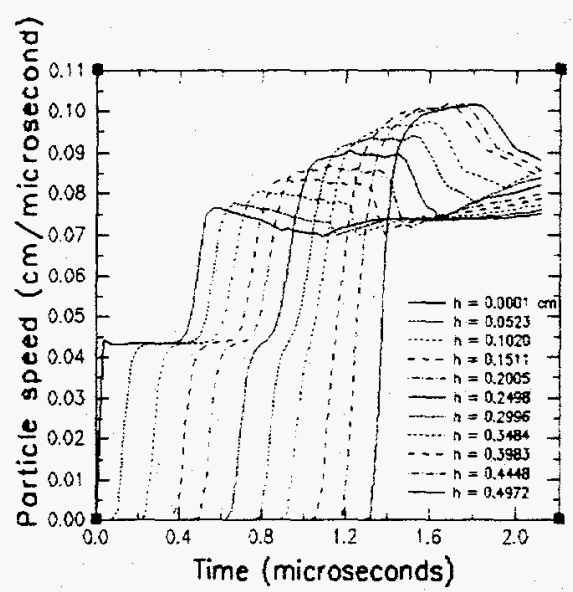

FIGURE 3. HYDROX-SCRAM CALCULATION OF MULTIPLE-SHOCK EXPERIMENT BY MULFORD ET AL.

The experimental results of Mulford are shown in Figure 2 and the HYDROX results are indicated in Figure 3. The agreement is fairly good, but improvements can be hoped for.

Three notable approximations are being addressed with more accurate treatments. First, the pressure of the burned products in the cracks is not currently combined with the SCRAM stresses, which would require a twofield analysis but, rather, the internal energy of the burned material is added to the internal energy in the equation of state to give an estimate of the energy in the mixed material, which influences the equation of state in the composite material. A more explicit treatment of the partial pressures due to burned gases is planned. Second, the burned gases are not applied to drive the cracks used in SCRAM, but drive a separate crack calculation that is a somewhat simplified treatment of crack behavior, but one that is appropriate for burning cracks. Third, a more complete treatment of unstable crack dynamics as a 2degree-of-freedom system with an internal pressure is in progress. The degrees of freedom are crack opening and crack radius, allowing for a detailed treatment with the method of generalized coordinates. Other refinements needed are: (1) feedback from the crack-heating calculation to the crack-mechanics calculation to account for the influence of melting on crack friction, which probably reduces the friction significantly; (2) the betadelta phase transition in HMX, which increases the material volume and changes the material behavior in an unknown way; (3) account for strain-rate effects in material behavior.

\section{CONCLUSIONS}

Many successful models of explosive reactions have been formulated, but they generally leave open a variety of parameters that are used to fit integral experiments. Though successful for vigorous reactions, in the low energy regime involved in XDT, non-shock initiation, the Stevens test. drop tests, cook-off tests, and other such experiments, those models have not had good success. The premise in SCRAM is that a more detailed treatment of hot spots is necessary, and we have pursued this concept by analyzing the behavior of cracks in general. and closed cracks as hot spots. This work has been supported in recent years by mechanical tests of explosives well into the strain-softening regime, which can be modeled with SCRAM. These results allow us to infer the number density of cracks, their average size, and their growth rate over a wide range of temperatures and strain rates. We have also been able to confirm the existence of large, nearly planar, inter-granular cracks following impact in the micrographs developed by Skidmore et $a^{19}$. On the reactive side, we have obtained models of ignition and burn rate from the work of Forest: Linan and Williams; and Ward, Son, and Brewster. These have been useful in representing the energy release in hot spots. These sources, plus a variety of other data described in the text, have allowed us to model reactions in explosives and propellants using mesoscopic models with parameters based on detailed experiments. The results tend to support the frictional crack model for hot spots, though detailed verification would be very difficult. The detailed behavior on the mesoscopic scale $(0.1$ to 1000 microns) may well be much more complex than accounted for in the current work, but we hope to have captured the main features. It would be good to have a more detailed idea of the role of phase transitions, bubble formation, twinning, plasticity, the composite character of PBXs, and numerous other details.

Originally, an integral approach to explosive detonation and sensitivity was taken by many researchers in which the process was outlined in a general way and 
certain constants were fit to integral experiments. With massive computer calculations now practical, a reductionist approach has become feasible, in which details at the molecular or grain level are simulated. Unfortunately the original (integral) approach does not provide any insight into the details of hot-spot formation, while the latter (reductionist) approach does not allow for simulations of complete systems. SCRAM takes an intermediate course by formulating a constitutive model that represents fracture and hot-spot formation by modeling the microstructure, but the computational burden is greatly reduced by considering ensembles of defects rather than their individual dynamics. This makes it possible to characterize the microstructure by fitting macroscopic data rather than analyzing the mesoscopic granular scale, about which very little is known. (Representing the effects of fines is an especially awkward necessity.) Defects can be treated with mesoscale degrees of freedom such as crack opening and growth, driven by internal pressure of the burn products and far-field stresses. This approach has proven very flexible in allowing physical effects to be added as new information becomes available.

\section{ACKNOWLEGEMENTS}

We are indebted to D.A. Wiegand for carrying out experiments that allowed us to fit the initial loading and strain-softening behavior of explosives, and to J. Aidun for organizing the data in electronic form and preliminary analysis. D. Mulford supplied us with the velocities for her multiple-shock experiment. Useful conversations with these authors are gratefully acknowledged. Financial support and encouragement were received from P.M. Howe.

\section{APPENDIX A, SUPERPOSITION OF PENNY- SHAPED CRACKS}

The additional compliance due to a distribution of penny-shaped compression cracks can be written as ${ }^{17}$

$$
c^{c}=\beta^{s} \sum(b-2 a) N_{c} c^{3} \Delta \Omega \Delta c
$$

Here the summation is taken over the cracks that shear under one-dimensional compressive-stress, and $\mathbf{a}$ and $\mathbf{b}$ are fabric tensors. Equation A.1 is a general relation for the four-index compliance, but for one-dimensional stress we need only consider a scalar relation and cracks at $45^{\circ}$ to the loading direction, for which the normal is

$$
\mathbf{n}=(1 / \sqrt{2}, 1 / \sqrt{2}, 0)
$$

Then $a_{1111}=1$ and $b_{111}=1 / 4$ are the only non-vanishing contributions to $C^{\alpha}$. The constant $\beta^{x}$ comes from the theory of penny-shaped cracks ${ }^{17}$ and is given by

$$
\beta^{s}=8(1-v) / 3 \mu(2-v)
$$

where $v$ denotes Poisson's ratio and $\mu$, the shear modulus.

The number of cracks exceeding $c$ in radius, denoted by $\mathbf{N}$, depends on crack size, orientation, and time, since the cracks grow with time. Thus, the number can be written as $N(c, \Omega, t))$. Its derivative $N_{c}$ gives the corresponding number density. A Liouville equation that governs the evolution of $\mathrm{N}$ is given by Dienes ${ }^{17}$. The summation of Eq. A.1 is taken over cracks that are not locked by friction or otherwise constrained. Cracks perpendicular or parallel to the loading direction do not deform in this model.

Rather than carry out a detailed crack analysis and summation, we resort to an approximation used in the SCRAM code. The continuous distribution of crack orientations is approximated with 9 discrete orientations (bins). Of that total, cracks with 4 orientations shear during uni-axial stress while the remaining 5 do not. Crack orientations range over $2 \pi$, half the unit sphere, so the $\Delta \Omega$ are taken to be equal to $2 \pi / 9$. Then the summation over all orientations indicated in Eq. A.1 is equivalent to multiplying by $8 \pi / 9$. Recall that $N$ is the number of cracks per $2 \pi$ per cc, so that the total number $\mathrm{N}$ of cracks per cc is $N=\sum N \Delta \Omega$. With this discretization of orientations the data fit described here can be used directly in SCRAM. Then,

$$
C_{111}^{c}=C^{c}=\frac{4 \pi \beta^{S}}{9} \int_{0}^{x} N_{c} c^{3} d c
$$

If the cracks all have the same size, $N$ is a Heaviside function and

$$
\mathrm{C}^{\mathrm{c}}=\frac{4 \pi \beta^{s}}{9} N_{0} \mathrm{c}^{3}
$$

where $N_{0}$ is the number of cracks per unit volume per $2 \pi$, which remains constant.

\section{APPENDIX B, THE EFFECT OF SLOW CRACK GROWTH ON FRACTURE AND INSTABILITY}

The classic theory of crack instability and growth assumes that cracks grow at high speed when the stress exceeds a critical level. Practical observations on a variety of materials reported by Stroh ${ }^{20}$ show that crack tip speeds can approach roughly a third the longitudinal speed of sound, while Freund ${ }^{21}$ claims on theoretical grounds that they approach the Rayleigh speed at high stresses, somewhat faster than the Stroh result. On the other hand, it has been observed ${ }^{22.23}$ that, when the stress is below the critical level for instability, cracks may grow at low speeds. (This regime may be governed by diffusion rather than dynamic processes.) The empirical result can be expressed as 


$$
v=v_{R}\left(K / K_{1}\right)^{n}
$$

This result can be supposed to apply when the stress is below a critical level. At high stresses Freund ${ }^{21}$ gives a complementary result

$$
\mathrm{v}=\mathrm{v}_{R}\left(1-\mathrm{K}_{0}^{2} / \mathrm{K}^{2}\right)
$$

The constants in $B .1$ can be estimated by forcing the magnitude and slope of the curves defined by B.1 and B.2 to agree at some $K$. to be determined. The constants in B. 2 are presumed known from the theory reported by Freund, with $K_{0}$ the critical value of the stress intensity factor and $v_{R}$ the Rayleigh speed. This agreement requires that

$$
1-K_{0}^{2} / K^{2}=\left(K / K_{1}\right)^{n}
$$

and

$$
2 K_{o} / K^{3}=n\left(K / K_{1}\right)^{n-1} / K_{1}
$$

Combining these equations leads to:

$$
K_{1}=K\left(n K^{2} / 2 K_{0}^{2}\right)^{1 / n}
$$

at the value of $K$ where intersection occurs, henceforth denoted by $K^{\prime}$. Combining this with B.3 leads to

$$
K^{\prime}=K_{0}(1+2 / n)^{1 / 2}
$$

Substituting this result back in B.5 results in the expression

$$
K_{1}=K_{0}(n / 2)^{1 / n}(1+2 / n)^{1 / 2+1 / n} \quad .
$$

For closed penny-shaped cracks, the configuration assumed in this report the critical condition is

$$
c_{c r}=\sqrt{\pi(2-v) \gamma \mu /(1-v)} /(\sigma-\tau)
$$

where $\gamma$ is the surface energy, $\mu$ is the shear modulus, $\sigma$ is the shear stress and $\tau$ is the resisting frictional stress. The corresponding fracture toughness is

$$
K_{0}=\sqrt{\pi c}(\sigma-\tau)=\pi \sqrt{(1-v / 2) \gamma \mu(1-v)}
$$

The relative crack speed at the cross-over point where $\mathrm{K}=\mathrm{K}^{\prime}$ is

$$
\mathrm{v} / \mathrm{v}_{0}=1 /(1+n / 2)
$$

which is $1 / 7$ for $n=12$, the value used in the optimization reported in the main text. In this kind of approach we no longer deal with crack instability, but with a transition from slow to fast growth as stress increases.

\section{APPENDIX C, MATERIAL PROPERTIES}

vistal kel-F PBX

9501

Density, gm/cc

$4.103 \quad 2.120$

1.860

Bulk Modulus, $\mathrm{Kb}$

$4.640 \quad 80.6$

$\mathrm{S},\left(d U_{s} / d U_{p}\right)$

1.87

2.26

Poisson Ratio

0.36

$\Gamma$, Gruneisen Ratio

Mean Crack Size, microns

Number Density, $\mathrm{cm}^{-3} / 2 \pi$

Gammal, ergs $/ \mathrm{cm}^{2}$ (HMX)

50

Gamma2, ergs $/ \mathrm{cm}^{2} \quad$ (PBX 9501)

308,000

\section{APPENDIX D, FRACTURE TOUGHNESS OF COMPOSITE}

The increase in surface energy can be explained by the following physical argument. Consider the binder to be distributed as a set of thin square tiles separating cubes of HMX of side $a$, whose individual volume is $a^{2} b$. Assume that the tiles have a failure energy of $\varepsilon \sigma$ per unit volume, where $\varepsilon$ is the strain to failure and $\sigma$ is the average flow stress. Now, the number of HMX cubes per unit volume is roughly $m=1 / a^{3}$ and the volume fraction $f$ of the binder is given. Since there are 3 tiles of binder per cube of HMX, $3 a^{2} b m=f$ for a large, random area (crack surface) the number of tiles intersected per unit area is $n=2 / a^{2}$. Then the energy per unit area consumed in braking the binder tiles is

$$
\gamma_{\mathrm{c}}=\mathrm{n} \varepsilon \sigma \mathrm{a}^{2} \mathrm{~b}=2 \varepsilon \sigma \mathrm{af} / 3
$$

With the rough estimates $\varepsilon=1.0, \sigma=40 \mathrm{MPa}, \mathrm{a}=100$ microns, binder volume $7.3 \%$ of the total ( $f=0.073$ ), the surface energy due to tearing of the binder is $\gamma_{\mathrm{c}}=195 \mathrm{~J} / \mathrm{m}^{2}$. This is sufficiently consistent with the value obtained from Wiegand's data, and well in excess of the surface energy of HMX.

\section{References}

'Jensen, R.C., Blommer, E.J., and Brown, B. "An Instrumented Shotgun Facility to Study Impact Initiated Explosive Reactions", in Seventh Symposium 
(International) on Detonation, Annapolis, MD. 16-19 June 1981, pp. 299-307

${ }^{2}$ Green, L. G., James E., Lee, E.L., Chambers, E.S., Tarver, C.M., Westmorland, C., Weston, A.M., and Brown, B. "Delayed Detonation in Propellants from Low Velocity Impact," in Proc. 7th Symposium on Detonation, J.M. Short, Ed., Annapolis, Md. June 16-19, 1981, pp. 254-264

${ }^{3}$ Mulford, R.N., Sheffield, S.A., and Alcon R.A., "Initiation of Preshocked High Explosives PBX 9404, PBX 9502, PBX 9501, Monitored with In-Material Magnetic Gauging," Tenth International Detonation Symposium, Boston, MA. 12-16 July 1993, pp. 459-467

${ }^{4}$ Von Holle, W.G., "Shock Wave Diagnostics by TimeResolved Infrared Radiometry and Non-linear Raman Spectroscopy," Shock Waves in Condensed Matter-1983, Elsevier Science Publishers, 1984, pp. 283-292

${ }^{5}$ Schmitt, D.R., Ahrens, T.J., and Svendsen, B., "Shockinduced melting and shear banding in single-crystal $\mathrm{NaCl}, " J G R$ 94, 85, May 10,1989, pp. 5851-5871

${ }^{6}$ Wiegand D.A., Private communication

'Dienes, J.K., "Strain-Softening via SCRAM" in preparation

${ }^{8}$ Dienes J.K., and Riley N., "Strain-Softening of PBX 9501, , in preparation

${ }^{9}$ Dienes, J.K. "Frictional Hot-Spots and Statistical Crack Mechanics," in Proc. 1983 Annual Meeting of the Materials Research Society, J.H. Crawford, Jr., Y. Chen and W.A. Sibley, eds. Boston, MA, Nov. 1983, pp. 373 381

${ }^{10}$ Linan, A. and Williams, F.A. "Theory of Ignition of a Reactive Solid by Constant Energy Flux," Combustion Science and Technology, Vol. 3, 1971, pp. 91-98

${ }^{11}$ Ward, M.J., Son, S.F., and Brewster, M.Q., "Steady Deflagration of HMX with Simple Kinetics: A New Modeling Paradigm," 35th Aerospace Sciences Meeting, Rieno, NV, Jan. 6-10 1997

${ }^{12}$ Boggs, T.L., "The Thermal Behavior of Cyclotrimethylene-trinitramine (RDX) and Cyclotetramethyleneteranitramine (HMX)," in Fundamentals of Solid-Propellant Combustion, (K.K. Kuo and M. Summerfield. Eds.), Progress in Astronautics and Aeronautics, AIAA, New York, 1984, pp.121-175

${ }^{13}$ Parr, T.P., Boggs, T.L., Price. C.F., and Parr, D.M., "Measurement of the Temperature Sensitivities of ANAPA and HMX Buming Rates," 19th JANNAF Combustion Meeting, CPIA Pub. 366, Vol. 1, Oct. 1982, pp.281-288

${ }^{14}$ Forest, C.A., "Burning and Detonation," Seventh Symposium (International) on Detonation, June 16-19, 1981, Annapolis, MD, pp. 234-243
${ }^{15}$ Reuss, A., "Berucksichtigung der elastischen Formanderung in der plastizitats Theorie," Zeits. Ang. Math. Mech., 10, 1930, pp. 266-269

${ }^{16}$ Dienes, J.K., "Foundations of Statistical Crack Mechanics," in International Symposium on Intense Dynamic Loading and Its Effects," Zheng Zhemin, chairman, Beijing, China, June 3-7 1986, Pergamon Press, pp. 602-607

${ }^{17}$ Dienes, J.K., “A Unified Theory of Flow, Hot Spots, and Fragmentation, with an Application to Explosive Sensitivity," in "High-pressure Shock Compression of Solids II", Davison, L., Grady, D.E., and Shahinpoor, M.. eds., Springer, 1996, pp. 366-398

${ }^{18}$ Sharma, J., Coffey. C.S., Ramaswamy, A.L. and Armstrong, R.W., "Atomic Force Microscopy of Hot Spots in Impacted RDX and Laser Heated AP," in Mat. Res. Symp. Proc., Eds. Brill, T.B., Russell, T.R., Tao, W.C., and Wardkle, R.B., 418, Materials Research Society, 1996

${ }^{19}$ Skidmore, C.B., Phillips, D., and Crane, N.B., "Microscopical Examination of Plastic-Bonded Explosives," Microscope, 45, 4, pp.127-136, 1997

${ }^{20}$ Stroh, A.N., "A Theory of the Fracture in Metals" in "Advances in Physics". N.F. Mott, Ed., Vol. VI, Nos. 2124, Taylor and Francis Ltd., London, 1957

${ }^{21}$ Freund, L.B., "Dynamic Fracture Mechanics". Cambridge University Press. 1990

${ }^{22}$ Evans, A.G., "Slow Crack Growth in Brittle Materials under Dynamic Loading Conditions," Int. J. Fracture, 10, 2, 1974, pp. $251-259$

${ }^{23}$ Charles, R.J., "Dynamic Fatigue of Glass," JAP 29, 12. 1958 , pp. 1652-1657

${ }^{24}$ Palmer, S.J.P. and Field, F.E., "The deformation and fracture of $\beta$-HMX." Proc. R. Soc. Lond. A, 383, 1982. pp. 399-407

${ }^{25}$ Bowden, F. P. and Yoffe, Y. D., "Initiation and growth of explosion in liquids and solids", Cambridge University Press, 1952

${ }^{26}$ Dienes, J.K.. Kershner J.D., "Multiple-Shock Initiation with Statistical Crack Mechanics" in preparation. (This is an expanded version of the current paper)

${ }^{27}$ Dienes, J.K. "A Frictional Hot-Spot Theory for Propellant Sensitivity." in Proc. 2nd JANNAF Propulsion Systems Hazards Meeting, China Lake. CA, April 19-21. 1982

${ }^{28}$ Shaw, M.S. and Straub, G.K. "HYDROX: A OneDimensional Lagrangian Hydrodynamics Code" Los Alamos National Laboratory report LA-8642-M Manual UC-32, Issued: March 1981 